\title{
Progress towards Quantitative Electron Nanodiffraction
}

\author{
J.M. Zuo, R. Twesten, B.Q. Li, J. Tao, Y.F. Shi, J. Bording, H. Chen and I. Petrov. \\ Department of Materials Science and Engineering and Materials Research Laboratory \\ 1304 W Green Street, Urbana, IL 61801
}

Here we report our recent progress toward quantitative electron nanodiffraction. Electron nanodiffraction using sub-nanometer probes was developed by Cowley, Brown and others in late 70's using VG -STEM [1]. Recent progress in FEG -TEM's has brought this capability to the reach of most materials research laboratories. Compared to VG-STEM, FEG-TEM offers a range of electron detectors and energy filtering, which makes quantitative study of electron nanodiffraction possible. There are two types of electron diffraction patterns that can be recorded with a nanometer -sized electron probe. One with a focused electron probe gives convergent beam electron diffraction (CBED). The other with a divergent, parallel, electron beam gives a selected-area-type diffraction. CBED patterns are routinely obtained in modern electron microscopes. In past ten years, tremendous progress has been made in developing CBED into a quantitative technique for strain measurements, symmetry determination and structure factor refinement [2-4]. The advantages of CBED are apparent: the diffraction pattern comes from a well-defined small volume; and there is rich information in CBED that comes from the parallel recording of diffraction intensities versus beam directions. All of these make CBED a useful technique for characterizing crystals. For non -crystalline materials, or crystals with limited sizes or shapes, the applicability of CBED is limited.

Electron nanodiffraction with a parallel beam is achieved by focusing the electron beam at the front focal plane of the objective pre -field. Under such condition, the size of illumination is determined by the size of condenser aperture (the spatial resolution of SAED is limited by $\mathrm{C}$ s). The smallest probe is obtained with an FEG-TEM with a small aperture. Fig. 1 shows an electron probe obtained from the JEOL2010FEG using NBD mode. It has a sharp peak of $1 \mathrm{~nm}$ half width and ripples from the coherence of electrons and aperture diffraction Fig. 2 shows an example of electron diffraction patter $\mathrm{n}$ using this probe from a nanometer -sized catalyst particles. The rich information in this pattern, a considerable improvement over the previous nanodiffraction patterns, underlies the potential of this technique for characterizing nanostructures. An impor tant application of electron nanodiffraction is characterizing the dynamics of nanostructures by diffuse scattering. Compared to $\mathrm{X}$-ray and neutron diffraction, electron small probe can be used to obtain single crystal diffraction patterns from complex materials that are diff icult to study otherwise. Fig. 3 shows an example of this [5]. Quantitative analysis of electron nanodiffraction requires 1) accurate measurement of electron diffraction intensities and 2) theoretical modeling. Experimentally, the advance in CCD cameras and electron energy -filter has made the first possible. Theoretical modeling is complicated by the nature of nanostructures and generally limited knowledge about these structures. A solution to this is to combine theoretical structure predictions with experimental measurements using techniques such as molecular dynamics and first -principle calculations. Progress in this area will be reported. In summary, recent progress in electron microscope instrumentation has made it possible to obtain quantitative electron nanodiffraction patterns with a parallel beam for recording both Bragg and diffuse scattering . Quantitative interpretation of these diffraction patterns requires a combination of structural modeling and ab-initio structure determination [6]. 
References:

[1] Techniques of Electron Diffraction, Edited by J. Cowley, Oxford Univ. Press (1992)

[2] K. Tsuda and M. Tanaka, Acta Cryst., A55 (1999) 939.

[3] L. Wu, Y. Zhu and J. Tafto, Phys. Rev. Lett. 85, (2000) 5126

[4] J.M. Zuo, M. Kim, M. O’keeffe, J. Spence, Nature, 401 (1999) 49.

[5] J. Zuo and J. Tao, Phys. Rev. B Rapid Comm., 63, 060407-1 (2001)

[6] This work is supported by DOE
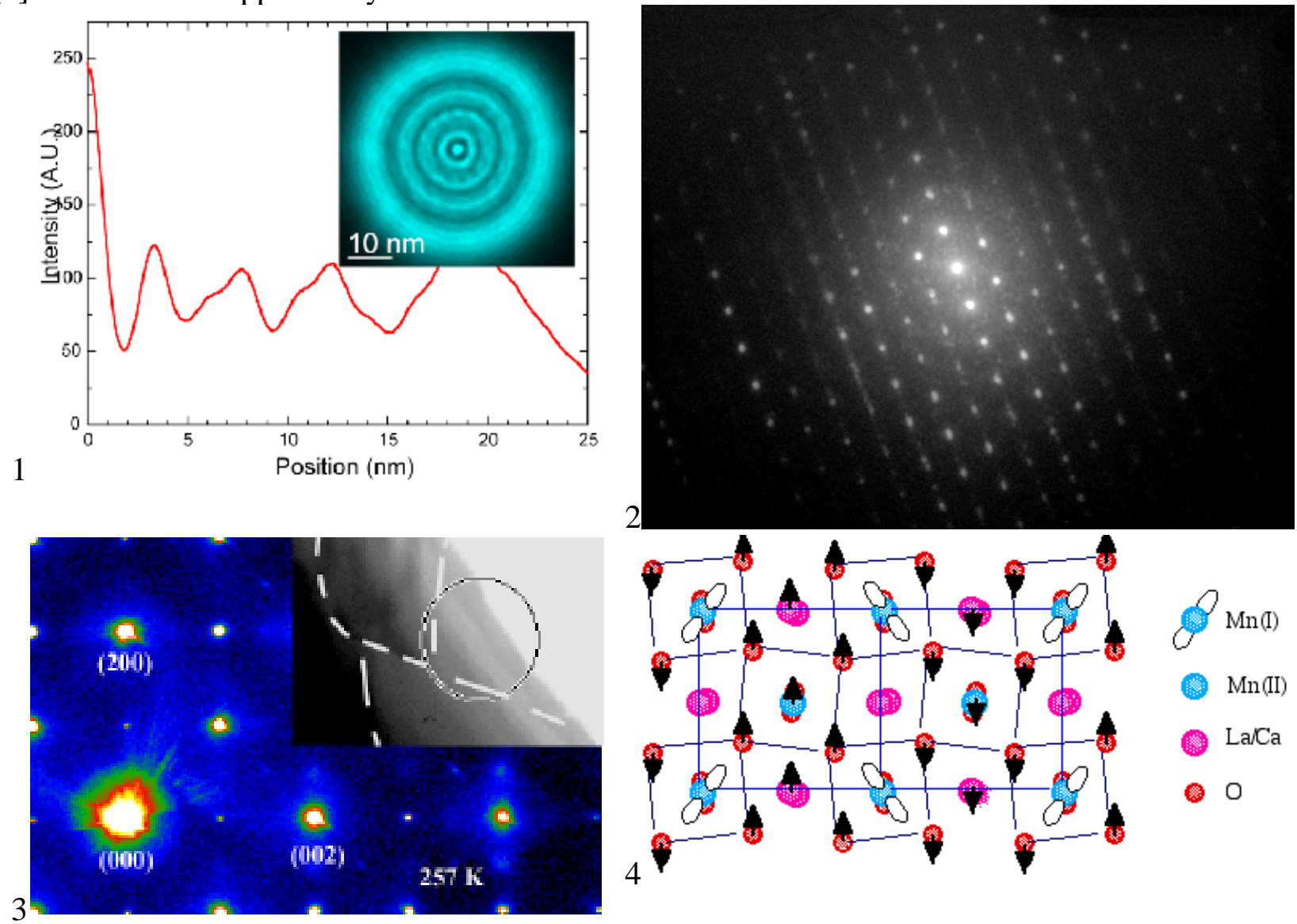

Fig. 1 Nanometer -sized electron probe formed with a parallel beam. Fig.2 Electron nanodiffractio $n$ of a catalytic particle. Fig. 3. Electron nanodiffraction of CMR oxides. Fig. 4 Structure model obtained from fig. 3. 